\title{
A Atenção Primária à Saúde e o enfrentamento à pandemia da COVID-19: um mapeamento das experiências brasileiras por meio da Iniciativa APS Forte
}

\author{
Michelle Fernandez, Wellington Carvalho, Vanessa Borges, Dirceu Klitzke, Renato Tasca
}

\section{RESUMO}

A Iniciativa APS Forte tem como objetivo principal identificar, reconhecer e divulgar boas práticas em Atenção Primária à Saúde que ocorrem no território brasileiro, sistematizando processos e transformando conhecimento tácito em explícito. A partir de uma parceria entre a OPAS/OMS e o Ministério da Saúde, busca-se dar visibilidade e promover iniciativas locais, municipais, regionais ou estaduais gerando conhecimento de forma ascendente e horizontal com vistas ao aumento da cooperação e solidariedade entre os trabalhadores, gestores, usuários e especialistas. 0 presente artigo tem como objetivo apresentar e descrever a Iniciativa APS Forte realizada em 2020, que foi dedicada a mapear o enfrentamento à pandemia da COVID-19 na APS no Brasil.

Palavras-chave: Atenção Primária à Saúde, COVID-19, Iniciativa APS Forte;

\section{ABSTRACT}

The "Strong PHC Initiative" has the main objective to identify, recognize and disseminate good practices in Primary Health Care (PHC) that occur in the Brazilian territory, systematizing processes and transforming tacit into explicit knowledge. Based on a partnership between PAHO/WHO and the Ministry of Health, the aim is to give visibility and promote local, municipal, regional, or state initiatives, generating knowledge in an ascending and horizontal manner, with a view to increase the cooperation and solidarity among workers, managers, users, and experts. This article aims to present and describe the Strong PHC Initiative conducted in 2020, which was dedicated to mapping the actions to face the COVID-19 pandemic in PHC in Brazil.

Keywords: Primary Health Care, COVID-19, Strong PHC Initiative.
Revista da Rede APS 2021

Publicada em: 28/12/2021

DOI:10.14295/aps.v3i3.216

Michelle Fernandez

(Universidade de Brasília, Brasília, DF, Brasil)

Wellington Carvalho (Organização Panamericana da Saúde / Organização Mundial da Saúde (OPAS/OMS), Brasília, DF, Brasil)

\section{Vanessa Borges}

(Organização Panamericana da Saúde / Organização Mundial da Saúde (OPAS/OMS), Brasília, DF, Brasil)

Dirceu Klitzke

(Ministério da Saúde, Brasília, DF, Brasil)

\section{Renato Tasca}

(Organização Panamericana da Saúde / Organização Mundial da Saúde (OPAS/OMS), Brasília, DF, Brasil)

Correspondência para: Michelle Fernandez michelle.vfernandez@gmail.com 


\section{INTRODUÇÃO}

O Brasil é internacionalmente reconhecido pelo seu sistema de saúde, que é público e universal. A Constituição Brasileira de 1988, conhecida como constituição cidadã, incorporou em seu capítulo da seguridade social a saúde como um direito de todos e dever do Estado. Em seguida, a Lei 8080/1990 criou o Sistema Único de Saúde (SUS), que foi regulamentada pelo Decreto 7508/2011 onde expressa que acesso ao sistema de saúde será ordenado pela Atenção Primária à Saúde (APS).

A Atenção Primária à Saúde é considerada a principal e mais adequada forma de acesso das pessoas ao sistema de saúde, estando diretamente associada a uma distribuição mais equitativa da saúde entre populações (Starfield \& Macinko, 2005). No Brasil, a APS tem se desenvolvido ao longo dos últimos 30 anos, com a implementação dos Agentes Comunitários de Saúde (ACS) em 1991, com a criação do Programa Saúde da Família (PSF) em 1994, que foi posteriormente consolidado na Estratégia Saúde da Família (ESF), e com a publicação da primeira Política Nacional de Atenção Básica em 2006. Assim, foram criados uma série de programas e estratégias para qualificar e expandir a APS, e possibilitar a consolidação desse modelo tecno-assistencial nos municípios brasileiros.

O novo arranjo institucional Brasileiro, pósConstituição de 1988, apontou para avanços importantes na direção da descentralização de políticas públicas no país, conferindo maior autonomia a estados e municípios. Nesse novo arranjo, os municípios ficaram responsáveis por organizar, executar e gerenciar os serviços e ações de Atenção Básica, de forma universal, dentro do seu território, incluindo as unidades próprias e as cedidas pelo estado e pela União (PNAB, 2017), entretanto há responsabilidades comuns as três esferas de governo. Nesse sentido, cada governo local tem a capacidade de desenvolver estratégicas próprias e inovadoras para ordenar e adaptar seus serviços ao enfrentamento dos desafios de saúde pública, utilizando da melhor forma possível os recursos disponíveis.
Em março de 2020, a Organização Mundial de Saúde (OMS) declarou o surto da COVID-19 como pandemia. Desde então, o Brasil e o mundo têm enfrentado a mais importante crise sanitária dessa geração. Observando as experiências de enfrentamento à pandemia pelo mundo, constata-se que aqueles países que centraram a resposta à COVID-19 na atenção hospitalar tiveram seus sistemas de saúde rapidamente colapsados. Dessa forma, fica claro que, a exemplo do que aconteceu em outras epidemias no Brasil e no Mundo (Miller et al., 2018; Dunlop et al., 2020), o enfrentamento comunitário é uma potente estratégia de atuação frente a COVID-19. E, nesse sentido, a Atenção Primária à Saúde cumpre um papel fundamental em função das suas características.

Assim, diante dos desafios gerados pela atual crise sanitária, países com APS consolidada e como porta de entrada do sistema de saúde têm a possibilidade de articular o combate à pandemia de forma eficaz. As ações dos entes locais são essenciais nessa resposta à crise sanitária vivida nos dias de hoje. Em países como - Brasil, com a ESF/APS sendo o serviço mais capilarizado da rede e organizada pelos governos locais, torna-se primordial conhecer e entender a diversidade de organização, reestruturação e atuação da Atenção Primária à Saúde no atual contexto se faz fundamental. Nesse sentido, mapear experiências é necessário para orientar estratégias inovadoras.

O termo "APS Forte" passou a ser utilizado pela representação da Organização Pan-Americana da Saúde (OPAS) no Brasil a partir do estudo realizado com gestores, pesquisadores e especialistas envolvidos com experiências significativas na APS no SUS. O estudo apontou um conjunto de 20 recomendações para a APS brasileira e tem servido como marco teórico estratégico para as ações de cooperação técnica no país (Tasca et al., 2018). Dentro dessas ações, a OPAS/OMS desenvolveu uma parceria com o Ministério da Saúde chamada "Iniciativa APS Forte". Seu objetivo principal é identificar, reconhecer e divulgar boas práticas em APS que ocorrem no território brasileiro, sistematizando processos e transformando conhecimento tácito em explícito. Busca-se dar visibilidade e 
promover iniciativas locais, municipais, regionais ou estaduais gerando conhecimento de forma ascendente e horizontal com vistas ao aumento da cooperação e solidariedade entre os trabalhadores, gestores, usuários e especialistas.

Nesse sentido, a Iniciativa em 2020 teve o título "APS Forte no SUS no combate à pandemia" e contou com 1670 experiências inscritas. Dessas, 1471 foram aprovadas. Em 2019 tivemos 12\% a menos de experiências aprovadas, ou seja, 1239 experiências. 26 Unidades da Federação tiveram experiências aprovadas na edição 2020 da Iniciativa APS Forte. Apenas o estado do Tocantins não teve experiências aprovadas nesta edição da Iniciativa. A realização da iniciativa, teve como objetivo identificar, reconhecer e dar visibilidade para promover a troca de experiências entre trabalhadores e gestores. Também manifestar apreço ao esforço e qualidade das equipes em promover iniciativas locais, municipais, regionais ou estaduais que tenham como foco a organização, melhoria e inovação da Atenção Primária à Saúde no contexto da pandemia de COVID-19. Nas linhas que seguem, apresentaremos um mapeamento qualitativa das iniciativas apresentadas.

\section{DESCRIÇÃO DA MATRIZ DE ANÁLISE E SUAS CATEGORIAS ANALÍTICAS}

A APS é um nível de atenção à saúde integrado e acessível, capaz de atender à grande maioria das necessidades em saúde das pessoas, desenvolvendo vínculos sustentáveis no contexto da família e da comunidade (Ellner \& Phillips, 2017; Epperly et al., 2019; Starfield, Shi \& Macinko, 2005). A OMS indica que os sistemas de saúde que têm uma estrutura baseada no trabalho colaborativo e em equipe na APS não apenas melhoram os resultados de saúde, mas também melhoram a equidade, apoiam a atenção integral e longitudinal (Ellner \& Phillips, 2017; Epperly et al., 2019; Starfield, Shi \& Macinko, 2005).
Os profissionais de atenção primária apontam as atividades de vigilância, o diagnóstico precoce, o tratamento de casos leves, a implementação de medidas de prevenção e educação em saúde como seu papel central durante uma pandemia (Clark, 2015; Hogg et al., 2006). Esses papéis e funções podem ser aprimorados dentro de um sistema de saúde integrado, funcionando com base na comunicação clara e no funcionamento de comitês de crise (Clark, 2015; Hogg et al., 2006). Em diferentes países da Europa, uma rede de vigilância de atenção primária foi implementada, demonstrando sua eficácia para a influenza sazonal (Deckers et al., 2006; Torner et al., 2019).

A APS em sistemas universais de saúde configura-se como o primeiro ponto de contato para os pacientes. Em contextos de emergência sanitária em virtude de epidemias, esse nível de atenção continua sendo o canal de acesso ao sistema de saúde mais importante para os usuários. Alguns exemplos são a epidemia de dengue na Malásia, em que $83,9 \%$ das pessoas com a doença foram atendidas pela APS antes de internações hospitalares; a pandemia de H1N1 no sudeste de Ontário, onde a APS foi responsável pela vigilância, administração de antirretrovirais e continuidade do cuidado (Ang et al., 2010; Wynn \& Moore, 2012). Nesse sentido, os profissionais da APS estão bemposicionados para fazer um diagnóstico precoce, identificar contatos e fornecer informações de qualidade durante uma crise sanitária em função do vínculo já estabelecido com a comunidade local (Ang et al., 2010; Deckers et al., 2006; Hogg et al., 2006).

A partir da discussão apresentada anteriormente, e com base na literatura especializada, descrevemos nas linhas que seguem a matriz de análise e suas respectivas categorias utilizada para analisar as experiências apresentadas na Iniciativa APS Forte 2020. A matriz de análise se baseia em cinco linhas de atuação da APS durante a pandemia de COVID19. Essas linhas foram definidas a partir de uma revisão bibliográfica detalhada e considerando as especificidades observadas para a pandemia de COVID-19 no Brasil (WHO, 2020; Fernandez \& 
Lotta, 2020; Clark, 2015; Hogg et al., 2006; Starfield, Shi \& Macinko, 2005; Engstrom, 2020).
Na tabela a seguir, estão elencadas as categorias e subcategorias utilizadas para a análise:

Quadro 1 - Descrição das categorias e subcategorias utilizadas na análise

\begin{tabular}{|c|c|}
\hline Categoria & Subcategoria \\
\hline \multirow{5}{*}{ Vigilância Epidemiológica } & Rastreamento de casos suspeitos \\
\hline & Monitoramento da pandemia \\
\hline & Rastreamento de contatos \\
\hline & Testagem na UBS \\
\hline & Testagem fora da UBS \\
\hline \multirow{2}{*}{ Monitoramento de casos } & Monitoramento de casos leves \\
\hline & Monitoramento de altas hospitalares \\
\hline \multirow{2}{*}{$\begin{array}{l}\text { Informação sobre cuidados e } \\
\text { prevenção }\end{array}$} & Medidas de prevenção \\
\hline & Educação em saúde (disseminação de informação) \\
\hline \multirow{4}{*}{$\begin{array}{c}\text { Manutenção dos princípios da } \\
\text { APS }\end{array}$} & Continuação do cuidado (pacientes crônicos/gestantes/crianças) \\
\hline & Coordenação do cuidado (integração com outros serviços) \\
\hline & Integralidade (acesso a outras queixas além de sintomas gripais, etc.) \\
\hline & Manutenção do acesso \\
\hline \multirow{9}{*}{$\begin{array}{c}\text { Atenção especial a grupos } \\
\text { vulneráveis }\end{array}$} & Gestantes \\
\hline & Idosos \\
\hline & População em situação de rua \\
\hline & Indígenas \\
\hline & Quilombolas \\
\hline & Pessoas com deficiência \\
\hline & Doentes crônicos \\
\hline & Migrantes \\
\hline & Pessoas em privação de liberdade \\
\hline \multirow{5}{*}{ Outros } & Reorganização do serviço \\
\hline & Saúde mental \\
\hline & Uso de novas tecnologias \\
\hline & Atenção ao profissional de saúde \\
\hline & Gestão de crise \\
\hline
\end{tabular}

Fonte: Elaboração própria.

Além das categorias vislumbradas pela literatura especializada, incluímos na matriz de análise a categoria "outros" e cinco subcategorias dentro dela. Essa categoria faz referência a outros temas não previstas inicialmente na análise, mas que chamaram a atenção ao longo da análise do material, seja pela quantidade de vezes que aparece, seja pela importância da temática para repensar a atuação da APS no atual contexto. Entre as subcategorias estão:

1. Reorganização do serviço: refere-se à necessidade de adaptar os serviços de saúde dado o contexto da pandemia. Essa subcategoria contempla novas escalas de trabalho, novas funções assumidas, novas formas de trabalho etc.;

2. Saúde mental: refere-se a experiências voltadas a questões ligadas à saúde mental, de forma geral;

3. Uso de novas tecnologias: uso de tecnologias diversas para teleatendimento e disseminação de informações;

4. Atenção ao profissional de saúde: observou-se um número expressivo de 
experiências voltadas especificamente para atender aos profissionais da saúde. Essa subcategoria contempla experiências que tem como foco a saúde física e mental desses profissionais e aquelas ações relacionadas com programas de residência e residentes; $\mathrm{e}$

5. Gestão de crise: faz referência ao uso de medidas para gerenciar a APS em tempos de pandemia, como o controle de medicamentos e insumos, redistribuição de recursos, profissionais e serviços etc.

\section{APRESENTAÇÃO DAS EXPERIÊNCIAS DA APS FORTE À LUZ DA MATRIZ DE ANÁLISE}

\section{Uma fotografia do conteúdo \\ analisado}

Começamos a análise das experiências aprovadas na Iniciativa APS Forte 2020 apresentando um retrato dos achados qualitativos do material enviado. Na tabela abaixo estão as palavras mais citadas na experiência, a frequência com a qual a referida palavra aparece, além da porcentagem de documentos que contém a palavra em questão. Em quase $100 \%$ das experiências aparece a palavra "Saúde" e em quase $90 \%$ aparece "Pandemia". Chama a atenção que pouco mais de $67 \%$ das experiências trazem a palavra "Profissionais", cerca de $62 \%$ apresentam a palavra "Equipe" e quase $51 \%$ contém a palavra "Social".

Em muitos relatos se observa um momento inicial de incertezas e da necessidade de readequação de processos e serviços realizados pelas equipes de saúde, para que se tornassem compatíveis com a nova realidade. Nesse sentido, muitas experiências (13\% delas) tem um caráter de gestão de crise e de reorganização dos serviços. É necessário chamar atenção para o fato de que as escalas de incidência das ações também se apresentam de forma distinta. Por um lado, observam-se experiências que nascem das UBS em si, voltadas para gerenciar a crise de distintas formas, bem como reorganizar seus serviços; por outro, observamos estratégias de gestão de secretarias de saúde municipais, com um foco mais extenso de reorganização e apoio às UBS e às equipes de saúde dos municípios.

Tabela 1 - Frequência de palavras a partir do conteúdo das experiências

\begin{tabular}{ccccc}
\hline Palavra & Frequência & $\%$ & Ranking & Experiência \% \\
\hline Saúde & 10250 & 2,41 & 1 & 97,35 \\
\hline COVID-19 & 4025 & 0,95 & 2 & 77,91 \\
\hline Pandemia & 3921 & 0,92 & 3 & 89,39 \\
\hline Profissionais & 3173 & 0,75 & 4 & 67,10 \\
\hline Atendimento & 2299 & 0,54 & 5 & 56,49 \\
\hline Equipe & 2287 & 0,54 & 6 & 62,27 \\
\hline Atenção & 2211 & 0,52 & 7 & 64,45 \\
\hline Pacientes & 2203 & 0,52 & 8 & 43,64 \\
\hline Ações & 2095 & 0,49 & 9 & 52,28 \\
\hline Casos & 2025 & 0,48 & 10 & 48,88 \\
\hline Usuários & 1917 & 0,45 & 11 & 46,30 \\
\hline População & 1894 & 0,45 & 12 & 53,98 \\
\hline
\end{tabular}




\begin{tabular}{ccccc}
\hline Cuidado & 1875 & 0,44 & 13 & 50,71 \\
\hline Trabalho & 1697 & 0,40 & 14 & 52,28 \\
\hline UBS & 1686 & 0,40 & 15 & 41,81 \\
\hline Unidade & 1631 & 0,38 & 16 & 46,16 \\
\hline Social & 1577 & 0,37 & 17 & 50,78 \\
\hline APS & 1453 & 0,34 & 20 & 34,19
\end{tabular}

Fonte: Elaboração própria.

Essa reorganização contempla duas dimensões: a primeira, de sentido mais prático, relaciona-se ao espaço físico das UBS, de modo a ordenar os atendimentos e separar os casos sintomáticos e/ou suspeitos daqueles de demandas que não estão relacionadas à síndrome gripal. A segunda dimensão diz respeito à adequação de serviços, em geral através do uso de tecnologias (teleatendimento, redes sociais, vídeos etc.) ou de uma reestruturação da forma de prestação dos serviços. Nesse sentido, compatível com as distintas atividades desenvolvidas pelas UBS no Brasil, a experiência de muitos profissionais foi levar suas especificidades para o mundo virtual ou adaptá-las como forma de promover atenção à saúde e conscientização da pandemia. No caso de nutricionistas há uma preocupação na educação sobre o manejo correto de alimentos e embalagens como forma de prevenção da COVID-19, sem deixar de lado as funções típicas destes profissionais. De maneira análoga, os profissionais de educação física buscaram desenvolver suas atividades de práticas corporais como forma de combate ao sedentarismo e a problemas de saúde mental decorrentes do isolamento social.

Essas duas linhas de atuação demonstram que existe a preocupação na APS em manter seus princípios, isto é, continuar prezando pelo cuidado aos pacientes e à comunidade a partir de uma readequação da forma de prestar serviço. Todas essas experiências demonstram que a manutenção do acesso, coordenação do cuidado e continuação do cuidado continuaram centrais nesse processo de readaptação das rotinas das UBS. Em 680 experiências (46,2\% delas) tem alguma menção à "Manutenção dos Princípios da APS".
A utilização de tecnologias diversas - tanto para teleatendimento e disseminação de informações, o uso de celulares, redes sociais e distribuição de mensagens é um traço marcante nas experiências em análise - foi a forma apresentada para dar continuidade ao funcionamento da APS em alguns sentidos. As palavras "WhatsApp", "Instagram", "Facebook", "YouTube", "redes sociais" e "internet" são citadas em 443 experiências (30,1\% delas), sendo que "WhatsApp" aparece em 306 destas $(20,8 \%)$. A prevalência pelo modo remoto pode ser observada pela menção deste tipo de atendimento em 626 experiências (42,5\%) citam pelo menos uma das palavras: "remoto", "telefone", "teleconsulta", "telemonitoramento" ou "teleatendimento".

Nesse contexto, chamou a atenção uma série de ações voltadas aos profissionais da saúde. Dentro das experiências, aparece a preocupação com a saúde mental desse grupo; 68 experiências específicas $(4,6 \%)$ sobre a questão. Apenas sobre "saúde mental", sem contemplar profissionais de saúde, há 266 experiências (31,8\%).

Outro tema que chamou a atenção numericamente foi a quantidade de menções à saúde da pessoa idosa. A palavra "idoso" é mencionada em 253 experiências (17,2\%), sendo o foco da abordagem em 137 destas (9,3\%). De maneira similar, grupos específicos como pacientes crônicos $(7,21 \%)$ e gestantes e puérperas $(3,87 \%)$ também foram priorizados pelas experiências aprovadas. Esses três grupos que representaram $28,28 \%$ das experiências expressam grupos que historicamente possuem 
oferta de ações programadas para esses usuários.

De forma geral, as ações de monitoramento de casos são ainda bastante incipientes e o tema da testagem é extremamente reduzido. Apenas $4 \%$ das experiências relatam testagem dentro da UBS e menos de $2 \%$ as mencionam de forma externa às unidades de saúde. $\mathrm{A}$ baixa testagem na APS é reflexo da ausência/insuficiência dessa estratégia no enfrentamento da pandemia. A APS sozinha não conseguiu reverter esse aspecto. Entretanto, cabe ressaltar que há um esforço de monitoramento da pandemia $(7,41 \%)$ geralmente com o objetivo de gerar boletins informativos para a atenção primária guiar as ações.

Gráfico 1 - Frequência de codificações em cada uma das categorias utilizadas

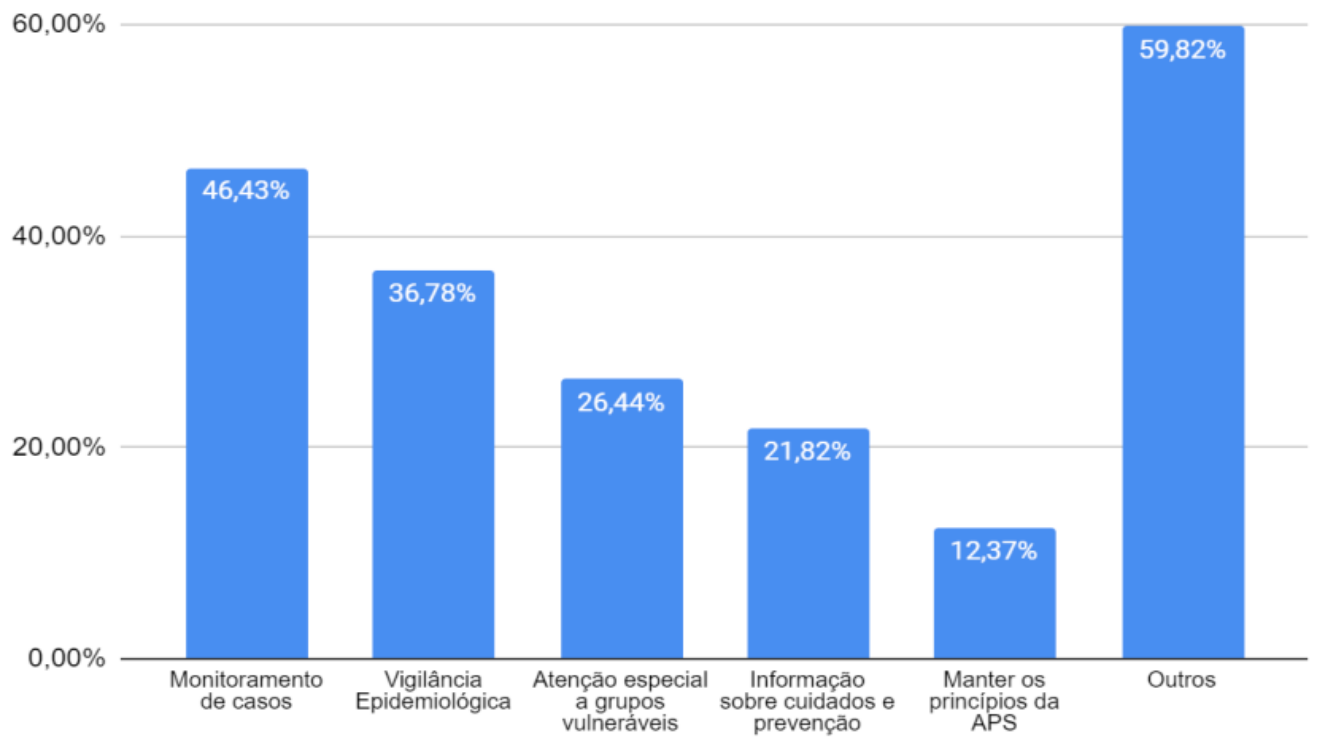

Fonte: Elaboração própria.

Destacamos nessa seção alguns resultados numéricos da análise qualitativa das experiências. No gráfico acima temos a frequência de codificações em cada uma das categorias utilizadas. Dado que as categorias não são mutuamente excludentes, a soma das porcentagens não é igual a $100 \%$. Isso se deve a que uma mesma experiência pode se referir a mais de uma categoria, compatível com as múltiplas atribuições da APS. Nesse sentido, observamos que $46 \%$ das experiências foram categorizadas em "Manter os princípios da APS".

\section{Algumas Ações da APS na pandemia contadas pelas experiências da APS}

Forte

As experiências apresentadas permitem que nos aproximemos das ações realizadas pela APS para enfrentar-se à crise sanitária vivida a partir da pandemia da COVID-19. A análise qualitativa aqui proposta se baseou na criação de categorias analíticas que serviram como instrumentos para ordenar e analisar os dados coletados. Para Minayo (2005), categorizar é agrupar elementos - ou ideias e expressões em torno de um único conceito, que é capaz de abranger todos estes elementos. Nesse sentido, nas linhas que seguem, apresentaremos exemplos, retirados das experiências, que nos 
mostram como cada categoria aparece no material coletado. Ao final de cada trecho identificamos o número que corresponde à experiência citada.

Os múltiplos caminhos e a diversidade de atuação da APS estão evidenciados na multiplicidade de categorias que as experiências analisadas buscam responder. As experiências apresentam classificações diversas, visto que também são múltiplas as necessidades de assistência à saúde na APS. Deste modo, observa-se que simultaneamente boa parte das ações se preocupa em promover uma busca ativa por casos e monitorar a pandemia ao passo em que promove orientações e disseminação de informações (educação em saúde), sem deixar de lado a manutenção dos princípios e diretrizes da APS.

Nas análises realizadas, chama a atenção que as experiências se voltam para uma reorganização do serviço motivada pelos desafios e pela nova realidade que surge no contexto da pandemia. Esta reorganização do ambiente pode ser observada na adequação de espaços físicos entre áreas de atendimento de casos suspeitos de COVID-19 e áreas para o público geral, por exemplo, conforme demonstram as experiências abaixo:

"A sala de recepção foi reorganizada para melhor otimização do espaço devido a necessidade (re)arranjo do fluxo de pacientes por conta da pandemia." (E. 1117)

"O processo de adequação das Unidades Básicas de Saúde (UBS) para atendimento a demanda com sintomas gripais se deu com a implantação de área de precaução (acolhimento, sala de isolamento, triagem, classificação de risco, consultório médico e sala de estabilização) para a identificação precoce dos sintomáticos gripais e sua classificação de risco. Foram elaborados fluxos de manejo clínico na APS $e$ instituído protocolos de tratamento." (E. 1436)
Observou-se ainda a necessidade de adequar os fluxos de trabalho e a prestação de serviços a partir da nova realidade trazida pela emergência sanitária. Nesse sentido, utiliza-se as novas tecnologias, focadas no atendimento à distância - teleatendimento, videochamadas, trocas de mensagens por aplicativos, entre outros - e o planejamento de atividades compatível com as normas de distanciamento social e para redução da circulação de pessoas nos espaços de saúde. Conforme apontam as Experiências abaixo:

\begin{abstract}
"No contexto da pandemia do novo coronavírus, os/as assistentes sociais, para se adequar às normas sanitárias, tiveram que reorganizar seu processo de trabalho delimitando quais atividades a serem mantidas e àquelas que deveriam ser temporariamente suspensas e/ou reformuladas." (E. 1384)
\end{abstract}

0 uso de tecnologias (telemedicina, telemonitoramento, WhatsApp e redes sociais) é central aqui, e se destaca como forma de facilitar o acesso de quem não pode mais ir até a Unidade Básica de Saúde ou para evitar idas desnecessárias em razão do risco de contaminação.

"A teleconsulta se apresenta como ponto fundamental para integralidade $e$ longitudinalidade e também é uma oportunidade de ampliação de acesso com segurança por reduzir a demanda presencial." (E. 1376)

"Uma das ações desenvolvidas, com vistas a apresentar saídas para o enfrentamento da crise, foi o uso do telemonitoramento em saúde. Implementado no final do mês de maio, este visa ampliar o acesso das pessoas aos pontos de atenção, por meio do uso da tecnologia, permitindo o atendimento remoto." (E.1251)

Além da utilização para monitoramento de pacientes - casos suspeitos e confirmados também são utilizados para a continuidade do cuidado, em especial os doentes crônicos e aqueles que mais demandam atenção, como gestantes, puérperas e recém-nascidos. 
Observa-se que o uso desta e de outras tecnologias como o WhatsApp permitiu a manutenção do acesso e também se configura como uma importante ferramenta, aliadas aos meios de comunicação tradicionais como TV e Rádio, para atividades de educação em saúde (informações sobre cuidado e prevenção).

"Esperançar' será um quadro
apresentado dentro do programa
"Guaiuba da Gente", na rádio
Comunitária Cultura FM 104.9. Esse
programa vai ao ar todas às segundas,
quartas e sextas, do horário de 12 às 14
horas e tem o apoio da prefeitura de
Guaiuba. A equipe da Secretaria de
Saúde tem usado o horário de 13 às 14
horas para divulgar os boletins
epidemiológicos e as informações
oficiais acerca da pandemia do novo
Coronavírus." (E. 383)

Com relação aos grupos vulneráveis, observa-se que os Idosos são os grupos com maior número de ações voltadas para sua atenção. Ilustramos essa preocupação nas experiências que seguem:

"Propor orientações para abordagem dos idosos pela APS, inseridas no contexto da Rede de Atenção à Saúde RAS COVID-19 MG, considerando que esta é uma subpopulação de risco para esta condição de saúde." (E. 414)

"Manter o idoso ativo nos períodos de quarentena da pandemia da Covid-19, levando até ele (residência) propostas de atividades e orientações para a saúde cognitiva, física e mental." (E. 1120)

As análises também demonstram uma preocupação especial com os próprios profissionais de saúde. Isto se justifica pela grande carga de serviços e de incertezas nesse novo contexto.

"Esse movimento exigiu grande dedicação dos profissionais da saúde, bem como uma sobrecarga de trabalho que gerou desgastes físicos $e$ emocionais. Na nossa equipe observamos aumento do estresse, relatos de medo de se contaminar ou de contaminar familiares, ansiedade diante $d a$ restrição da circulação social $e$ incertezas quanto ao futuro. Tal situação exigiu um movimento de cuidado aos profissionais de saúde da UBS e a equipe multidisciplinar foi convocada para iniciar oferta de Práticas Integrativas $e$ Complementares (PICS) para todos os trabalhadores que tivessem interesse nesse cuidado." (E. 1178)

Os desafios em busca de novas formas de manter o acesso dos pacientes aos serviços de saúde, em sua totalidade, se colocam como ponto central no cenário atual. Deste modo, observa-se que a manutenção dos princípios da APS se coloca como ponto chave para compreender a forma como as equipes de saúde têm lidado com este contexto. Neste sentido, as experiências estão próximas do relato:

\begin{abstract}
"Diversas estratégias foram pensadas como forma de reorientar o trabalho nas Unidades Básicas de Saúde durante a pandemia como adequação da estrutura física para garantir acesso seguro, proteção dos trabalhadores de saúde, fortalecimento da abordagem familiar e comunitária e manutenção do distanciamento social, fundamental medida preventiva à nova doença. $\mathrm{O}$ uso das tecnologias de comunicação pode ser um grande aliado nesse processo, mantendo a comunicação efetiva e mais frequente entre a unidade e os usuários e reduzindo deslocamentos pelo território." (E. 1225)
\end{abstract}

\section{CONCLUSÕES}

No contexto da pandemia de COVID-19, quando o setor saúde é desafiado para reorganizar os serviços de saúde para proteger a população, a troca de conhecimento e vivências se torna uma alternativa essencial para os profissionais de saúde e para os gestores. Em um país de dimensões continentais como o Brasil e muito heterogêneo, permitir o intercambio amplo em tempo oportuno também é um desafio, assim que estratégias que permitam a troca de 
conhecimentos de forma célere com clareza de conteúdo são fundamentais.

Os profissionais e gestores da APS brasileira que participaram da Iniciativa aqui analisada mostraram grande compromisso, resiliência, adaptação e capacidade de inovação, liderando processos, dentro do seu âmbito de atuação, para efetivar processos de reorganização dos serviços de Atenção Primária na resposta ao enfrentamento da pandemia, ao mesmo tempo que demonstraram preocupação com a continuidade dos cuidados e serviços essenciais.

A Iniciativa demonstra que, além dos resultados em termos de disseminação de conhecimento, as experiências divulgadas têm um efeito de superação e motivação, se tornando um reconhecimento da qualidade do trabalho da equipe responsável, constituindo, portanto, um estímulo para todos os profissionais comprometidos ("se o outro fez, eu também posso"). Entretanto, também fica patente a pouca institucionalidade na maioria das experiências apresentadas, mostrando que houve um vazio de normativas, políticas e programas desenhados com a singularidade que cada momento de enfrentamento da pandemia exigia para apoiar/orientar a resposta da APS à pandemia. Espera-se que 0 trabalho desenvolvido aqui apoie a tomada de decisão dos gestores públicos em construir políticas e programas de saúde em resposta a emergências de saúde pública orientadas pela APS.

Cabe aqui, saudar e agradecer a trabalhadoras e trabalhadores da APS brasileira que, no maior desafio de saúde pública da nossa história recente, demostraram seu compromisso genuíno em participar do esforço do Sistema Único de Saúde para enfrentar e superar a pandemia. 


\section{REFERÊNCIAS}

ANG, K. T., ROHANI, I., \& LOOK, C. H. Role of primary care providers in dengue prevention and control in the community. Medical Journal of Malaysia. 2010; 65(1), 58-62.

BARDIN, L. Análise de conteúdo. 2011; São Paulo: Edição 70.

CLARK, S. J. (). Role of primary care providers in a pandemic - conflicting views and future opportunities. Israel Journal of Health Policy Research. 2015; 4(1), 3-5. https://doi.org/10.1186/s13584-015-0054-3

DECKERS, J. G. M., PAGET, W. J., SCHELLEVIS, F. G., \& FLEMING, D. M. European primary care surveillance networks: Their structure and operation. Family Practice. 2006; 23(2), 151-158. https://doi.org/10.1093/fampra/cmi118

ENGSTROM, E. et. al. Recomendações para a organização da APS no SUS no enfrentamento da Covid-19. Observatório Covid-19 - Fiocruz. Maio/2020.

ELLNER, A. L., \& PHILLIPS, R. S. The Coming Primary Care Revolution. Journal of General Internal Medicine. 2017; 32(4), 380-386. https://doi.org/10.1007/s11606-016-3944-3

EPPERLY, T., BECHTEL, C., SWEENEY, R., GREINER, A., GRUMBACH, K., SCHILZ, J., ... O'CONNOR, M. The shared principles of primary care: A multistakeholder initiative to find a common voice. Family Medicine. 2019; 51(2), 179-184.

FERNANDEZ, M., \& LOTTA, G. Atenção básica e profissionais de saúde: a ponta de lança no combate à epidemia do Covid-19. Jornal Estado de São Paulo - Blogs. 2020; Acesso em 30 de outubro de 2021. https://politica.estadao.com.br/blogs/gestao-politica-e-sociedade/atencao-basica-e-profissionais-desaude-a-ponta-de-lanca-no-combate-a-epidemia-do-covid-19/

HOGG, W., HUSTON, P., MARTIN, C., \& SOTO, E. Enhancing public health response to respiratory epidemics. Canadian Family Physician. 2006; 52(10), 1254-1260.

MINAYO, M. C. D. S. Avaliação por Triangulação de Métodos. Abordagem de Programas Sociais. Rio de Janeiro: FIOCRUZ, 2005. https://doi.org/10.7476/9788575415474

STARFIELD, B., SHI, L., \& MACINKO, J. Contribution of Primary Care to Health Systems and Health. Johns Hopkins Bloomberg School of Public Health. The Milbank Quarterly. 2005; 83(3), 457-502.

TASCA R, MASSUDA A, CARVALHO WM, BUCHWEITZ C, HARZHEIM E. Recomendações para o fortalecimento da atenção primária à saúde no Brasil. Rev Panam Salud Publica. 2020; 44:e4. https://doi.org/10.26633/RPSP.2020.4

TORNER, N., BASILE, L., MARTÍNEZ, A., RIUS, C., GODOY, P., JANÉ, M., ... FERRÚS, G. Assessment of two complementary influenza surveillance systems: Sentinel primary care influenza-like illness versus severe hospitalized laboratory-confirmed influenza using the moving epidemic method. BMC Public Health. 2019; 19(1), 1-10. https://doi.org/10.1186/s12889-019-7414-9

WHO. (2020). Strengthening and Adjusting Public Health Measures throughout the COVID-19 Transition Phases. Copenhagen: WHO European Region.

WYNN, A., \& MOORE, K. M. Integration of primary health care and public health during a public health emergency. American Journal of Public Health. 2012; 102(11), 9-12. https://doi.org/10.2105/AJPH.2012.300957 